\title{
Ecology of stone-encrusting organisms in the Greenland Sea-a review
}

Piotr Kuklinski

Institute of Oceanology, Polish Academy of Sciences, Sopot, ul. Powstańców Warszawy 55, Sopot PL-81-712, Poland

\author{
Keywords \\ ecology; Greenland Sea; lithologic substrata; \\ lithophilic organisms; stones.

\section{Correspondence} \\ Piotr Kuklinski, Institute of Oceanology, Polish \\ Academy of Sciences, ul. Powstańców \\ Warszawy 55, Sopot PL-81-712, Poland \\ E-mail: kuki@iopan.gda.pl
}

doi:10.1111/j.1751-8369.2009.00105.x

\begin{abstract}
The Arctic is one of the most rapidly warming regions on Earth. This area is therefore very suitable for conducting studies focused on the influence of climate change on the biota. Marine communities of coastal waters are particularly sensitive to the current environmental changes (e.g., ice-scour intensity); understanding how community structure changes in response to local perturbations is thus important for providing an insight into how future communities may respond to climate change. This review focuses on the fauna colonizing stones of the Greenland Sea. It summarizes the current state of knowledge about the ecology of organisms inhabiting these substrata across a range of depths, from the intertidal to deeper parts of the continental shelf. In the intertidal zone, no stable or developed assemblage on the rocks is visible. The intertidal zone seems to be fully controlled by physical forces. In contrast, below the intertidal zone a rich and abundant fauna starts to appear on these substrata. Both biotic (e.g., competitive interactions) and abiotic (e.g., ice scour, size of the rock) processes seem to shape stone assemblages in the subtidal zone, yet their influence varies with depth. For example, the abundance of encrusting organisms decreases with depth, as does the intensity of competitive interactions. However, species richness on rocks seems to be in general higher in the deeper parts of the shelf. Possible scenarios of climate change influence on the encrusting biota, gaps in our knowledge about the ecology of stonedwelling faunal assemblages, as well as possible directions of future research, are discussed.
\end{abstract}

The last major glaciation affecting the Arctic began approximately 18000 years ago, and ended 6000 years ago (Dunton 1992). Ice sheets covered much of the Arctic continental shelf, and only the present areas of the Chukchi, Beaufort and East Siberian seas remained largely unglaciated, and almost entirely emergent. As a result, recolonization of many parts of the Arctic shelf by benthic fauna and flora has occurred over the last 6000-14 000 years (Dunton 1992). At present, benthic communities are composed of relatively young immigrants of Pacific and Atlantic affinity, and there are few endemics (Dunton 1992). The Arctic is considered to be a geologically young environment, still being colonized by marine organisms from lower latitudes (Dunton 1992). This polar region has repeatedly cooled slowly and warmed quickly in successive glacial cycles. Presently, the Arctic is warming more abruptly than at any previously recorded time (Schiermeier 2007). The effects of climate change in some parts of the Arctic are magnified in comparison with other polar regions. This is the case for the Greenland Sea, where the rapidity of the response results from the strong influence of the northward transport of warm Atlantic water (e.g., Loeng 1991; Cottier et al. 2005). The recent reappearance of the blue mussel (Mytilus edulis L.) in the eastern part of the Greenland Sea (on the coast of west Spitsbergen) after an absence of 1000 years is claimed to be a direct response to the current increase in sea-surface temperatures (Berge et al. 2005). Fluxes of warm Atlantic water masses into the same region have caused the appearance of temperate planktonic diatoms, and the mass occurrence of Atlantic cod (Gadus morhua L.) and Atlantic salmon (Salmo salar L.) (Berge et al. 2005).

Arctic coastal waters are probably the most sensitive of all habitats to environmental change. Ice, wave action and other disturbances mean that the polar coastline is in 
an almost perpetual state of recolonization. Therefore, understanding how community structure changes in response to local perturbations is particularly important for providing insights into how future communities may respond to climate change (Barnes \& Conlan 2007).

Coastal stones are common habitats for marine invertebrates across the globe, at all latitudes, providing the potential for multiple scale comparisons. They occur at all depths, from the intertidal zone to abyssal depths; however, they are most common near the coastline. The stones themselves come from different sources. Some are generated by processes of marine erosion or are transported by rivers (e.g., Reineck \& Singh 1975; Dowdeswell et al. 1998), whereas others have ended up on the sea floor because of changes in sea level that resulted in the flooding of coastal areas (Reineck \& Singh 1975). However, in marine polar regions, stones are most likely to be of glacial origin and are termed dropstones (Dowdeswell et al. 1998). Glaciers pick up rocks as they move across the land, incorporating them into the ice. As they reach the ice shelf, they break up into icebergs, which release their load of rocks into the sea when they melt, often after travelling long distances (Dowdeswell et al. 1998). In the polar regions, stones can additionally be moved from the littoral to subtidal zones by marine algae, which incorporate them into their holdfast, and are able to transport them by floating or dragging them over a large distance (Gilbert 1984).

In marine polar regions, rocks provide a habitat for many organisms, ranging from single-celled foraminifera to more complex sponges, ascidians, barnacles and polychaetes, and colonial organisms such as bryozoans and hydrozoans, the colonies of which often act as habitats for many other species (e.g., Barnes \& Kuklinski 2003; Kuklinski $\delta$ Bader 2007a). These firm substrata often offer unique opportunities for hard substratum biota to colonize part of the sea floor, which is otherwise blanketed by soft sediments (e.g., Oschmann 1990; Mayer \& Piepenburg 1996; Kuklinski \& Bader 2007a, b).

Stones often remain on the sea bottom for a long period of time, and are exposed to various biological colonizations and interactions. Yet, the colonization of hard substrata in the Arctic is about an order of magnitude poorer in diversity than that described from lower latitudes (Clarke \& Lidgard 2000). However, on the local scale, at some sites species richness can be as high, or higher than, that found in many similar habitats in the North Atlantic or Pacific (Clarke \& Lidgard 2000; Barnes \& Kuklinski 2005a). These coastal stones and their associated fauna thus provide a fantastic natural "laboratory" for testing various hypotheses, including those relating to competitive interactions (Barnes 2002; Barnes \& Kuklinski 2003), latitudinal patterns of diversity and abundance
(Kuklinsi, Barnes et al. 2006) and the intermediate disturbance hypothesis (Connell 1978).

There are several advantages to using the assemblages that encrust stones to explore benthic ecology. These include: (1) the wide availability of the substrata, and its easy access for sample collection; (2) the diversity and abundance of organisms that inhabit stones; (3) most taxonomic identifications are possible long after the death of the organisms; and (4) many of the keystone species have good fossil records, enabling studies over larger time scales (e.g., Sousa 1979; Wilson 1985, 1987; McGuinnes 1987; Barnes 2002; Taylor \& Wilson 2003; Kuklinski, Barnes et al. 2006).

The aim of this study is to review the current state of our knowledge about the ecology of organisms inhabiting stones (including pebbles, cobbles and boulders; adopting the Wentworth scale) in the Arctic, one of the globe's most rapidly changing environments. It focuses on western Spitsbergen and East Greenland (Fig. 1), where the majority of Arctic studies have been conducted. This compilation can serve as a baseline for future studies. Gaps in our knowledge, as well as possible future directions, are also discussed.

\section{Depth distribution of fauna associated with stones}

Several studies have indicated that there is a natural division of assemblages of stones in the Greenland Sea, expressed in terms of their species composition, and corresponding to three levels of zonations: intertidal, shallow subtidal (from extreme low tide down to 40-50 $\mathrm{m}$ in depth) and deep subtidal (from 40-50 $\mathrm{m}$ in depth to the edge of the continental shelf) (e.g., Kuklinski 2002; Kuklinski et al. 2005). Physical factors related to depth are most likely to be responsible for the pattern observed (Kuklinski et al. 2005). The intertidal zone is heavily battered by waves and scoured by ice. It is biologically impoverished, and seems to be fully controlled by physical factors (e.g., Weslawski et al. 1993, Barnes \& Kuklinski 2004b, Ronowicz 2005). In addition to physical forces (e.g., rock overturning), subtidal stone assemblages are also influenced by biotic factors (e.g., competition). Abiotic factors include strong currents generated by wind and iceberg scouring (recorded to a depth of $40 \mathrm{~m}$ at Kongsfjorden, western Spitsbergen [Dowdeswell \& Forsberg 1992]), which decrease in intensity with depth. Food availability seems to be greater in the shallow subtidal zone than in the deeper parts of the Greenland Sea, as does the abundance of biota (Booth \& Smith 1997; Hop et al. 2002). As a consequence, biological interactions (e.g., competition and predation) are probably more intense in the shallow subtidal zone than in deeper parts 


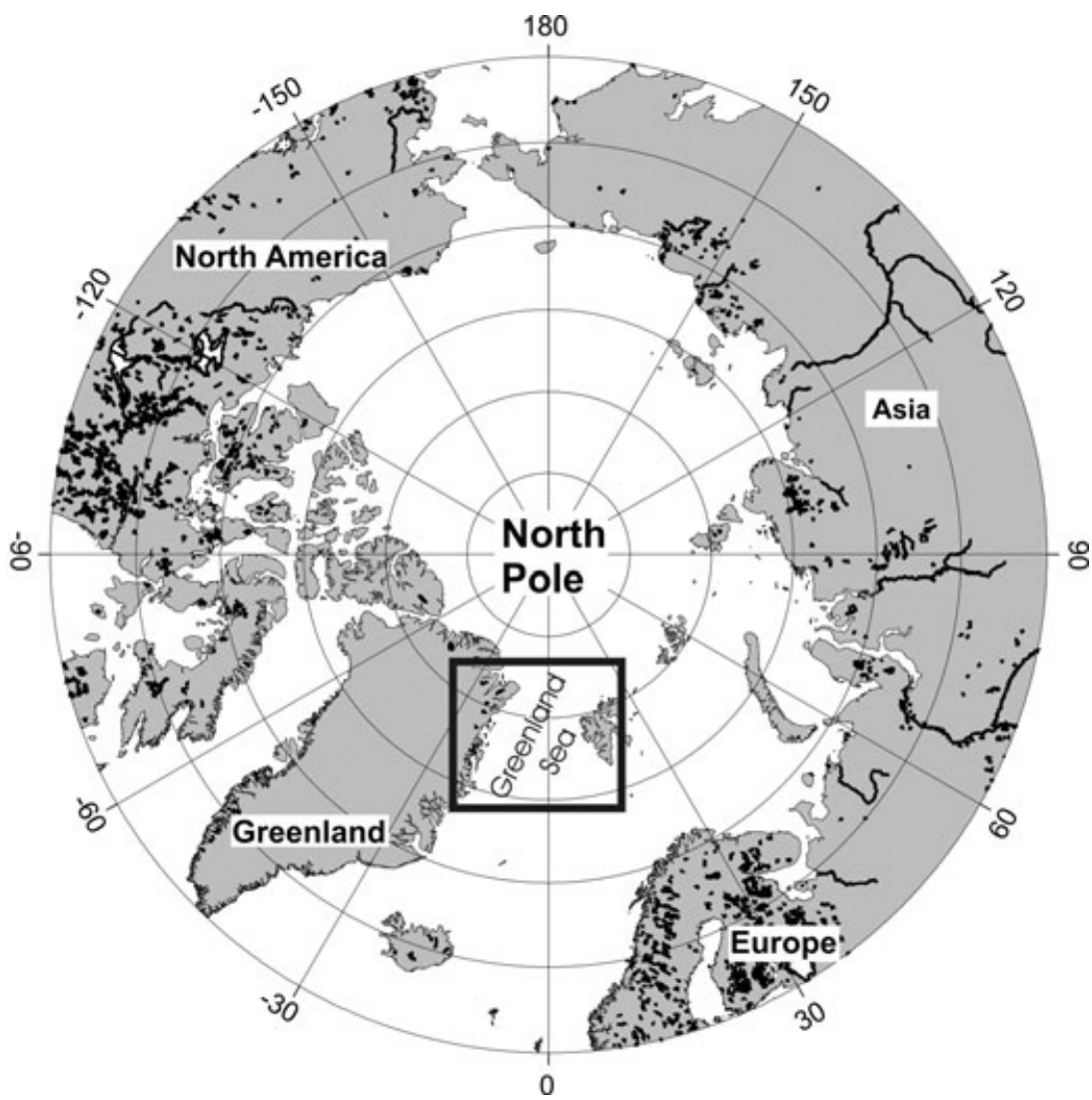

Fig. 1 Map of the showing the location of the Greenland Sea, the area covered by this review. of the shelf. The following sections focus on the three depth zonations (intertidal, shallow subtidal and deep subtidal).

\section{Intertidal zone}

Arctic coastal waters are probably one of the Earth's most physically disturbed environments, experiencing not just diurnal changes in sea level, but also seasonal changes in temperature, and the repeated freezing and thawing of the overlying water (Fig. 2). There is also intense summer ice scour, vigorous wave action and freshwater run-off, coupled with freezing temperatures and the formation of ice feet (narrow strips of ice that are frozen to the shore of many Arctic beaches) during the winter (Barnes 1999) (Fig. 2). Therefore, organisms inhabiting stones in this zone are exposed to extreme mechanical and thermal disturbances.

No stable or well-developed macroscopic assemblage is visible in the Arctic intertidal zone, either on rocks or other substrata (Weslawski et al. 1993; Szymelfenig et al. 1995; Kuklinski \& Barnes 2005a; Ronowicz 2005). The development of assemblages is very limited, even on the most stable or protected substrata. Fauna on intertidal stones have little time to develop beyond the pioneer stage, so these substrata continually remain in the early stages of colonization (Kuklinski, Barnes et al. 2006). The colonization of stones in the intertidal zone is very haphazard, and the majority of organisms that occur here do not reach reproductive age. The assemblage structure often mirrors the current or recent species abundances in meroplankton in the water column. Assemblages are dominated by those species that happen to settle in the highest abundance (species with the largest reproductive output) following the most recent disturbance event. High levels of disturbance in the Arctic are also reflected in the apparent lack of intertidal specialists. Intertidal stone colonizers are largely sourced from more stable subtidal populations nearby (Kuklinski \& Barnes 2005a; Kuklinski, Barnes et al. 2006; Kuklinski \& Barnes 2008). The frequency of catastrophic disturbance is high, and therefore the most abundant species are generally those with fast growth rates, early sexual reproductive capabilities and good powers of dispersal (the so called $r$-selected organisms; Begon et al. 1996).

Faunal assemblages of stones, if present, seem to be dominated by one or two species. For example, on the west coast of Spitsbergen the dominant taxa are the cirriped barnacle Semibalanus balanoides L. and the cheilostome bryozoan Harmeria scutulata Busk, but 
Fig. 2 Photographs illustrating some of the physical factors that shape the intertidal stoneencrusting fauna of western Spitsbergen. (a) The formation of an ice foot in the intertidal zone (a narrow strip of ice that is frozen to the shore of many Arctic beaches); early spring. (b) The intertidal zone disturbed by an ice foot during early spring; example from the Hornsund fjord in the vicinity of the Polish Polar Station. (c) The intertidal zone in Isfjorden showing the boulder field battered by wave action during the Arctic summer. (d) Small pieces of glacier ice (growlers) in the intertidal zone that often have a detrimental influence on the stone-encrusting fauna; summer, Kongsfjorden.
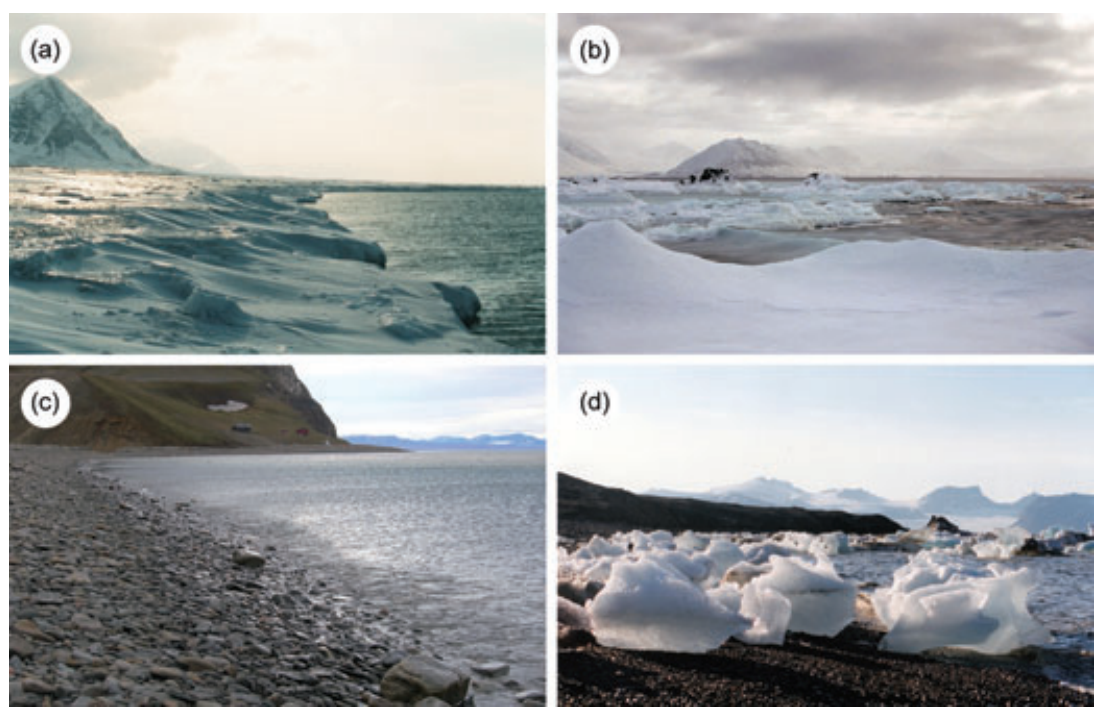

(d)

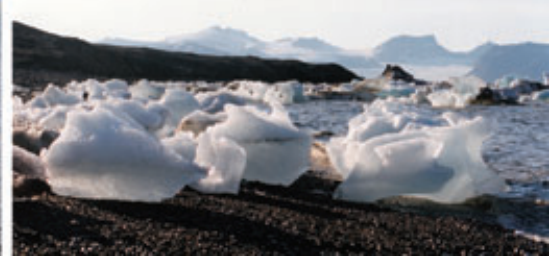

foraminifera and hydroids also occur (Kuklinski \& Barnes 2008). Harmeria scutulata comprised $57 \%$ of all individuals at six investigated locations on the west coast of Spitsbergen (Kuklinski \& Barnes 2005a, b). Greenland Sea intertidal assemblages on stones have very low diversities when compared with nearby subtidal boulder fields, and also with similar habitats from lower latitudes (Kuklinski, Barnes et al. 2006; Kuklinski \& Barnes 2008). The species richness from six similar locations in western Spitsbergen ranged from three to seven taxa per site, with a total of 17 taxa found across all of the investigated locations (Kuklinski \& Barnes 2008). The abundance of macrobiota in intertidal assemblages is also low (mean 496.5 individuals per $\mathrm{m}^{2}$ of the rocks surface per site), and is usually an order of magnitude lower than in the subtidal zone (mean 6267.6 individuals per $\mathrm{m}^{2}$ of the rocks surface per site [Kuklinski \& Barnes 2008]).

With such little life on the rocks in the intertidal zone, no competitive interactions have been found to date on stones (Barnes \& Kuklinski 2004a). The ice-mediated disturbance in the polar intertidal zone is so high that communities are void of competition for space (Dayton 1990; Arntz et al. 1994; Kuklinski \& Barnes 2005a). The Arctic intertidal zone seems to be fully controlled by physical forces.

\section{Shallow subtidal zone}

Below the intertidal zone, rich and abundant life starts to appear on the stones (Fig. 3; Table 1). However, most often the entire top surfaces of rocks are colonized by calcareous algae, which outcompete the majority of other organisms (Fig. 4). Therefore, the development of fauna in the shallowest subtidal zones takes place almost

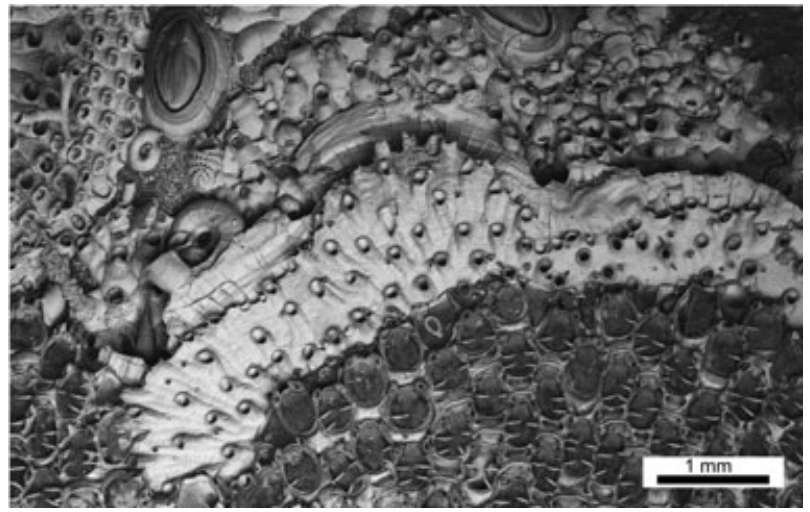

Fig. 3 Scanning electron microscope image of a small area of rock showing a high density of biota, including polychaetes, bryozoans and barnacles.

entirely on the undersurface of the stones (Barnes $\delta$ Kuklinski 2003). In the deeper subtidal regions the light intensity is lower, and calcareous algae are not able to develop. Additionally, at these depths soft sediment is more common, so colonization is almost exclusively on the top surface of the stones (Barnes \& Kuklinski 2003). The size of the stone is important for faunal development in the shallow subtidal zone. Recruitment is positively correlated $\left(r^{2}=0.96, P<0.001\right)$ with substratum size (Barnes \& Kuklinski 2003). The probability of fauna being present on stones of surface area less than $5 \mathrm{~cm}^{2}$ is nearly zero, whereas on stones larger than $100 \mathrm{~cm}^{2}$ the probability of finding some life is extremely high-ca. 100\% (Barnes \& Kuklinski 2003). Stone size also affects assemblage structure. At a depth of $10 \mathrm{~m}$, Kuklinski, Gulliksen et al. (2006) found that small stones $\left(<5 \mathrm{~cm}^{2}\right)$ support different species assemblages than larger stones. The 
Table 1 Taxa occurring on the stones in the Greenland Sea listed with their distribution, depth range and morphology. Abbreviations: A, endemic to the Arctic; EG, East Greenland; WS, west Spitsbergen it, intertidal. Data compiled from Barnes \& Kuklinski (2003, 2004b), Kuklinski \& Barnes (2005a), Kuklinski et al. (2005), Kuklinski, Gulliksen et al. (2006), Kuklinski \& Bader (2007a, b), Ronowicz (2007) and Kuklinski (unpubl. data).

\begin{tabular}{|c|c|c|}
\hline Taxa & $\begin{array}{l}\text { Location and depth } \\
\text { range }(\mathrm{m})\end{array}$ & Morphology \\
\hline \multicolumn{3}{|l|}{ FORAMINIFERA } \\
\hline Cibicides lobatulus (Walker \& Jacob, 1798) & WS (6-12) & Flat, encrusting \\
\hline Cassidulina reniforme (Norvang, 1945) & WS (6-12) & Flat, encrusting \\
\hline Elphidium sp. & WS (6-12) & Flat, encrusting \\
\hline Foraminifera indet. & EG (75-232) & Flat, encrusting \\
\hline \multicolumn{3}{|l|}{ PORIFERA } \\
\hline Leucosolenia complicata (Montagu, 1818) & WS (6-12) & Erect, flexible \\
\hline Grantia compressa (Fabricius, 1780) & WS (6-12) & Erect, flexible \\
\hline Scypha ciliata (Fabricius, 1780) & WS (6-12) & Erect, flexible \\
\hline Halichondria sp. & WS (6-12) & \\
\hline Haliclona sp. & WS (6-12) & \\
\hline Porifera indet. & EG (97-232) & \\
\hline \multicolumn{3}{|l|}{ CNIDARIA } \\
\hline Abietinaria pulchra (Nutting, 1904) & WS (79-115) & Erect, flexible \\
\hline Calycella syringa (L., 1758) & WS (5-117) & Erect, flexible \\
\hline Eudendruim ramosum (L., 1758) & WS (10) & Erect, flexible \\
\hline Gonothyrea loveni (Allman, 1859) & WS (5-111) & Erect, flexible \\
\hline Halecium sp. & WS $(5-10)$ & Erect, flexible \\
\hline Lafoea dumosa (Fleming, 1820) & WS (5-219) & Erect, flexible \\
\hline Lafoeina maxima Levinsen, 1893 & WS (94-111) & Erect, flexible \\
\hline Rhizocaulus verticillatus (L., 1758) & WS (79-108) & Erect, flexible \\
\hline Urticina felina (L., 1761) & WS (6-12) & Erect, flexible \\
\hline Hydrozoa indet. & EG (152-232) & Erect, flexible \\
\hline \multicolumn{3}{|l|}{ POLYCHAETES } \\
\hline Pomatoceros sp. & WS (6-12) & Flat, encrusting \\
\hline Serpulidae indet. & EG (75-232) & \\
\hline Bushiella quadrangularis (Stimpson, 1854) & WS (6-12) & Flat, encrusting \\
\hline Circeis spirillum (L., 1758) & WS (6-12) & Flat, encrusting \\
\hline Paradexiospira cancellata (Fabricius, 1780) & WS (6-12) & Flat, encrusting \\
\hline Paradexiospira vitrea (Fabricius, 1780) & WS (6-12) & Flat, encrusting \\
\hline Spirorbis spirorbis (L., 1758) & WS (6-12) & Flat, encrusting \\
\hline Spirorbis sp. & EG (75-232), WS (6-12) & Flat, encrusting \\
\hline Sabellidae indet. & EG (97-232) & \\
\hline \multicolumn{3}{|l|}{ CRUSTACEA } \\
\hline Balanus balanus (L., 1758) & WS (6-12) & Erect, rigid \\
\hline Balanus crenatus (Brugiere, 1789) & WS (6-12) & Erect, rigid \\
\hline Semibalanus balanoides (L., 1767) & WS (6-12) & Erect, rigid \\
\hline Balanus sp. & EG (75-221) & Erect, rigid \\
\hline \multicolumn{3}{|l|}{ CHORDATA } \\
\hline Botryllus sp. & WS (6-12) & Erect, flexible \\
\hline Diplosoma sp. & WS (6-12) & Erect, flexible \\
\hline Dendrodoa sp. & WS (6-12) & Erect, flexible \\
\hline \multicolumn{3}{|l|}{ BRYOZOA } \\
\hline "Proboscina sp." & EG (75-232), WS (12-220) & Flat, encrusting \\
\hline Alcyonidium erectum Andersson, 1902 (A) & EG (152-168) & Erect, membranous \\
\hline Alcyonidium sp. & WS (6) & Erect, membranous \\
\hline Amphiblestrum septentrionalis (Kluge, 1906) & EG (75-221) & Flat, encrusting \\
\hline Amphiblestrum trifolium (Wood, 1844) & WS (122) & Flat, encrusting \\
\hline Berenica arctica Kluge, 1946 (A) & EG (186) & Flat, encrusting \\
\hline Bowerbankia sp. & EG (186) & Erect, membranous \\
\hline Buffonellaria arctica Berning \& Kuklinski 2008 & WS (122-153) & Flat, encrusting \\
\hline Callopora aurita (Hincks, 1877) & WS $(6-12)$ & Flat, encrusting \\
\hline Callopora craticula (Alder, 1857) & EG (75-221), WS (it-226) & Flat, encrusting \\
\hline Callopora lata (Kluge,1907) (A) & WS (6-12) & Flat, encrusting \\
\hline Callopora cf. lineata (L., 1767) & WS (it-12) & Flat, encrusting \\
\hline Callopora smitti Kluge, 1946 (A) & WS (6-122) & Flat, encrusting \\
\hline
\end{tabular}


Table 1 Continued

\begin{tabular}{|c|c|c|}
\hline Taxa & $\begin{array}{l}\text { Location and depth } \\
\text { range }(\mathrm{m})\end{array}$ & Morphology \\
\hline Callopora weslawski Kuklinski and Taylor, 2006 (A) & EG (75-232) & Flat, encrusting \\
\hline Carbacea carbacea (Ellis and Solander, 1786) & WS (6-12) & Erect, flexible \\
\hline Cauloramphus cymbeaformis (Hincks, 1877) (A) & WS (12) & Flat, encrusting \\
\hline Cauloramphus intermedius Kluge, 1955 (A) & WS (6-12) & Flat, encrusting \\
\hline Cellepora nodulosa Lorenz, 1886 (A) & EG (108-221) & Erect, rigid \\
\hline Cellepora surcularis (Packard, 1863) (A) & EG (75-83) & Erect, rigid \\
\hline Celleporella hyalina (L., 1767) & WS (it-12) & Flat, encrusting \\
\hline Cheilopora sincera (Smitt, 1868) & EG (164-221), WS (6) & Flat, encrusting \\
\hline Cribrilina annulata (Fabricius, 1780) & WS (it-12) & Flat, encrusting \\
\hline Cribrilina spitsbergensis Norman, 1903 (A) & EG (205-221), WS (122) & Flat, encrusting \\
\hline Crisia sp. & EG (75-232), WS (it-6) & Erect, flexible \\
\hline Crisiella cf. producta (Smitt, 1865) & WS (6) & Erect, flexible \\
\hline Crisiella sp. & EG (97-221), WS (6-12) & Erect, flexible \\
\hline Cylindroporella tubulosa (Norman, 1868) & EG (75-106), WS (6-260) & Flat, encrusting \\
\hline Defrancia lucernaria Sars, 1851 & EG (152-221) & Erect, rigid \\
\hline Defrancia prolifera Kluge, 1946 & EG (75-83) & Erect, rigid \\
\hline Dendrobeania fruticosa (Packard, 1863) & WS (6-12) & Erect, flexible \\
\hline Dendrobeania murrayana (Johnston, 1847) & EG (75-83), WS (6-12) & Erect, flexible \\
\hline Dendrobeania pseudolevinseni Kluge, 1952 (A) & WS (12) & Erect, flexible \\
\hline Diplosolen arctica (Waters, 1904) (A) & EG (186) & Flat, encrusting \\
\hline Diplosolen intricarius (Smitt, 1872) (A) & EG (108-221) & Erect, rigid \\
\hline Diplosolen obelia (Johnston, 1838) & EG (108-221) & Flat, encrusting \\
\hline Disporella hispida (Fleming, 1828) & EG (97-186), WS (6-153) & Flat, encrusting \\
\hline Disporella sp. & EG (75-186), WS (6-260) & Flat, encrusting \\
\hline Doryporella spathulifera (Smitt, 1868) & EG (97-106), WS (12-260) & Flat, encrusting \\
\hline Electra arctica Borg, 1931 & EG (75-83), WS (6-153) & Flat, encrusting \\
\hline Electra catenularia-similis Kluge, 1962 & WS (122-153) & Flat, encrusting \\
\hline Entalophora clavata (Busk, 1859) & EG (186) & Erect, rigid \\
\hline Escharella abyssicola (Norman, 1869) & EG (75-232), WS (122) & Flat, encrusting \\
\hline Escharella klugei (Hayward, 1979) & EG (75-232), WS (6-12) & Flat, encrusting \\
\hline Escharella laqueta (Norman, 1864) & EG (152-169) & Flat, encrusting \\
\hline Escharella macrodonta Levinsen, 1916 (A) & EG (168-186) & Flat, encrusting \\
\hline Escharella ventricosa (Hassal, 1842) & EG (75-232), WS (6-122) & Flat, encrusting \\
\hline Escharelloides spinulifera (Hincks, 1889) (A) & WS (122-153) & Flat, encrusting \\
\hline Escharelloides stenostoma (Smitt, 1872) (A) & EG (108-232) & Flat, encrusting \\
\hline Escharoides bidenkapi (Kluge, 1946) & EG (152-232) & Flat, encrusting \\
\hline Escharoides jacksoni (Waters, 1900) (A) & EG (75-221) & Flat, encrusting \\
\hline Eucratea arctica (Kluge, 1915) (A) & EG (75-232) & Erect, flexible \\
\hline Eucratea loricata (L., 1758) & WS (it-12) & Erect, flexible \\
\hline Exidmonea atlantica Forbes, 1847 & EG (152-221) & Flat, encrusting \\
\hline Flustrellaria whiteavesi Norman, 1903 (A) & WS (12) & Flat, encrusting \\
\hline Harmeria scutulata (Busk, 1855) (A) & WS (it-12) & Flat, encrusting \\
\hline Hemicyclopora emucronata (Smitt, 1872) (A) & EG (97-186), WS (260) & Flat, encrusting \\
\hline Hippodiplosia borealis (Waters, 1900) (A) & EG (75-221) & Flat, encrusting \\
\hline Hippodiplosia harmsworthi (Waters, 1900) (A) & EG (75-232) & Flat, encrusting \\
\hline Hippodiplosia murdochi Kluge, 1962 (A) & WS (12) & Flat, encrusting \\
\hline Hippodiplosia obesa (Waters, 1900) & WS (12-260) & Flat, encrusting \\
\hline Hippodiplosia ussowi (Kluge, 1908) (A) & WS (12) & Flat, encrusting \\
\hline Hippothoa arctica Kluge, 1906 & EG (75-169), WS (6-260) & Flat, encrusting \\
\hline Hippothoa expansa Dawson, 1859 & WS (122-153) & Flat, encrusting \\
\hline Hornera lichenoides (L., 1758) & EG (108-232) & Erect, rigid \\
\hline Lepralioides nordlandica (Nordgaard, 1905) & EG (75-106), WS (122-260) & Flat, encrusting \\
\hline Microporella arctica Norman, 1903 (A) & WS (6-12) & Flat, encrusting \\
\hline Microporella klugei Kuklinski and Taylor, 2008 (A) & WS (122-153) & Flat, encrusting \\
\hline Notoplites smitti (Norman, 1903) (A) & EG (164-221) & Erect, flexible \\
\hline Oncousoecia canadensis Osburn, 1933 (A) & EG (75-232), WS(12-260) & Flat, encrusting \\
\hline Oncousoecia diastroporides (Norman, 1869) & WS(12-260) & Flat, encrusting \\
\hline Palmicellaria bicornis (Busk, 1859) & WS (122) & Erect, rigid \\
\hline
\end{tabular}


Table 1 Continued

\begin{tabular}{|c|c|c|}
\hline Taxa & $\begin{array}{l}\text { Location and depth } \\
\text { range }(\mathrm{m})\end{array}$ & Morphology \\
\hline Palmicellaria tridens (Busk, 1856) (A) & EG (108-221), WS (220) & Erect, rigid \\
\hline Parasmittina cf. trispinosa (Johnston, 1838) & WS (122-260) & Flat, encrusting \\
\hline Smittina rigida Lorenz, 1886 (A) & WS (6-12) & Flat, encrusting \\
\hline Phylactella labiata (Smitt, 1868) & EG (108-221) & Flat, encrusting \\
\hline Porella acutirostris Smitt, 1868 & EG (186), WS (122) & Flat, encrusting \\
\hline Porella aperta (Boeck, 1862) (A) & EG (108-232) & Flat, encrusting \\
\hline Porella leavis (Fleming, 1828) & EG (75-83) & Erect, rigid \\
\hline Porella minuta (Norman,1869) & WS (6-12) & Flat, encrusting \\
\hline Porella plana Hincks, 1888 (A) & EG (75-232) & Erect, rigid \\
\hline Porella princeps Norman, 1903 & WS (122-153) & Flat, encrusting \\
\hline Porella proboscidea Hincks, 1888 (A) & EG (75-221), WS (122-220) & Flat, encrusting \\
\hline Porella saccata (Busk, 1856) & WS (145) & Erect, rigid \\
\hline Pseudoflustra hincksi Kluge, 1915 (A) & EG (108-232) & Erect, flexible \\
\hline Pseudoflustra solida (Stimpson, 1854) (A) & EG (164-221) & Erect, flexible \\
\hline Ragionula rosacea (Busk, 1856) & EG (75-83) & Erect, rigid \\
\hline Reteporella beaniana (King, 1846) & EG (108) & Erect, rigid \\
\hline Reteporella cellulosa (L., 1758) & EG (186) & Erect, rigid \\
\hline Reteporella elongata Smitt, 1868 & EG (168-221) & Erect, rigid \\
\hline Reteporella watersi Nordgaard, 1907 (A) & EG (108-186) & Erect, rigid \\
\hline Rhamphostomella bilaminata (Hincks, 1877) (A) & WS (6) & Flat, encrusting \\
\hline Rhamphostomella costata Lorenz, 1886 (A) & WS (122) & Flat, encrusting \\
\hline Rhamphostomella ovata (Smitt, 1868) (A) & WS (6-12) & Flat, encrusting \\
\hline Rhamphostomella scabra (Fabricius, 1780) & EG (75-221), WS (122) & Flat, encrusting \\
\hline Rhamphostomella sibirica Kluge, 1929 (A) & WS (6) & Flat, encrusting \\
\hline Sarsiflustra abyssicola (Sars, 1872) & EG (164-221) & Erect, flexible \\
\hline Schizomavella lineata (Nordgaard, 1896) & WS (6-122) & Flat, encrusting \\
\hline Schizoporella costata Kluge, 1962 & WS (122-153) & Flat, encrusting \\
\hline Schizoporella crustacea (Smitt, 1868) (A) & WS (6-12) & Flat, encrusting \\
\hline Schizoporella elmwoodiae Waters, 1900 (A) & EG (75-232) & Flat, encrusting \\
\hline Schizoporella incerta Kluge, 1929 & WS (220) & Flat, encrusting \\
\hline Schizoporella limbata Lorenz, 1886 & EG (97-106) & Flat, encrusting \\
\hline Schizoporella pachystega Kluge, 1929 & EG (75-106), WS (12-260) & Flat, encrusting \\
\hline Schizoporella porifera (Smitt, 1868) & WS (12-260) & Flat, encrusting \\
\hline Schizoporella stylifera (Levinsen, 1887) & EG (75-106), WS (150-260) & Flat, encrusting \\
\hline Scrupocellaria arctica (Busk, 1855) (A) & WS (6-12) & Erect, flexible \\
\hline Septentriopora karasi Kuklinski and Taylor, 2006 & WS (6-12) & Flat, encrusting \\
\hline Smittina cf. belli (Dawson, 1859) & EG (75-221), WS (12-153) & Flat, encrusting \\
\hline Smittina glaciata (Waters, 1900) (A) & EG (186) & Flat, encrusting \\
\hline Smittina jeffreysi Norman, 1903 & EG (75-221) & Flat, encrusting \\
\hline Smittina majuscula (Smitt, 1868) & EG (186-221), WS (12) & Flat, encrusting \\
\hline Smittina minuscula (Smitt, 1868) & EG (75-186), WS (6-145) & Flat, encrusting \\
\hline Smittina peristomata (Nordgaard, 1905) & EG (108-232) & Flat, encrusting \\
\hline Prenantia bella Busk, 1860 & EG (75-186), WS (122-220) & Flat, encrusting \\
\hline Smittoidea exilis Hayward, 1994 & EG (108-221) & Flat, encrusting \\
\hline Stomachetosella cruenta (Busk, 1854) & EG (75-106), WS (6-260) & Flat, encrusting \\
\hline Stomachetosella producta (Packard, 1863) (A) & EG (75-221), WS (122-150) & Flat, encrusting \\
\hline Stomachetosella sinuosa (Busk, 1860) & EG (75-83), WS (150) & Flat, encrusting \\
\hline Stomatopora cf. granulata (Milne-Edwards, 1838) & EG (75-186) & Flat, encrusting \\
\hline Tegella arctica (d’Orbigny, 1850) & WS (it-122) & Flat, encrusting \\
\hline Tegella armifera (Hincks, 1880) (A) & WS (6-12) & Flat, encrusting \\
\hline Tegella armiferoides Kluge, 1955 (A) & EG (108-186), WS (122) & Flat, encrusting \\
\hline Tegella cf. retroversa Kluge, 1952 (A) & WS $(6-12)$ & Flat, encrusting \\
\hline Tricelaria ternata (Ellis and Solander, 1786) & WS (it-12) & Erect, flexible \\
\hline Tubulipora flabellaris (Fabicius, 1780) & WS (6-12) & Flat, encrusting \\
\hline Umbonula arctica (Sars, 1851) & WS (6-12) & Flat, encrusting \\
\hline
\end{tabular}




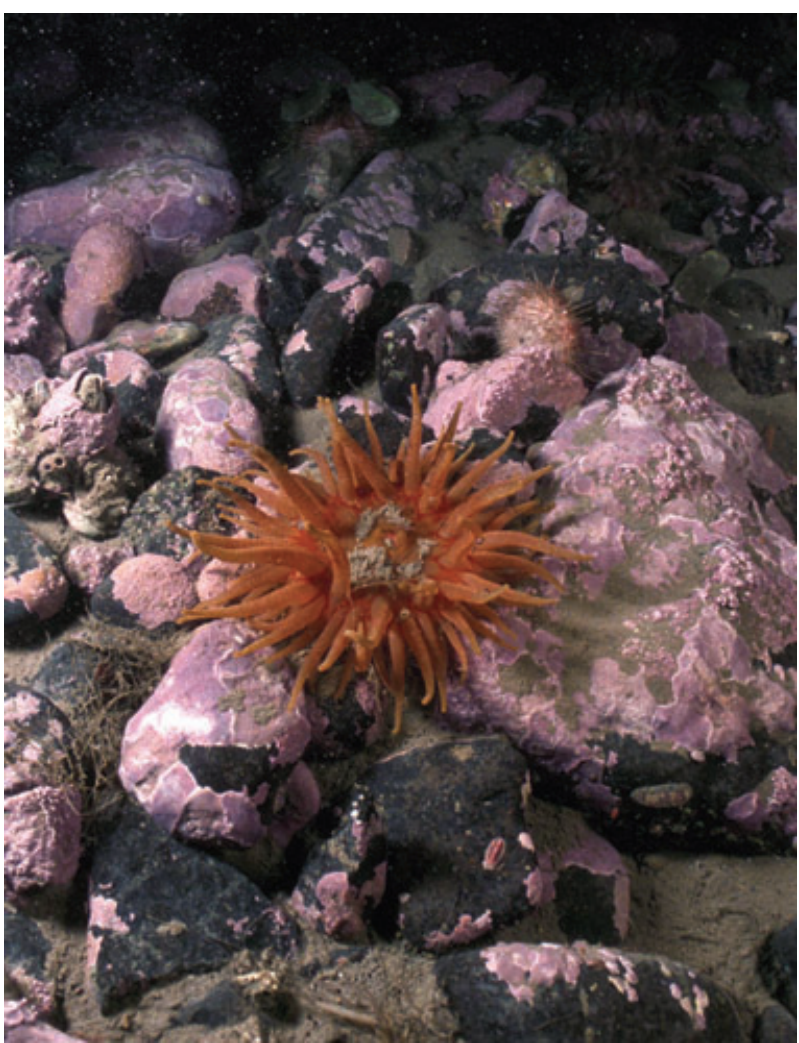

Fig. 4 Shallow subtidal stones from a depth of $12 \mathrm{~m}$. The upper surface is heavily overgrown by calcareous algae that have outcompeted other encrusting fauna.

fauna hosted by small stones $\left(<2 \mathrm{~cm}^{2}\right)$ or rocks from shallower depths is probably recruited from larvae present in the water column at the time of colonization (Kuklinski \& Barnes 2008). The fauna that inhabits small stones is in a state of constant transformation. Any physical force like strong wave action caused by storms will overturn small rocks more frequently than larger stones. Organisms that settle on such a dynamic substratum can develop only until the next disturbance (Kuklinski, Gulliksen et al. 2006). In this sense, small stones are similar to the stones in the intertidal zone. The assemblages growing on these small stones resemble the assemblages found in the intertidal zone. Not many organisms are able to survive under such unpredictable conditions: those that do are chiefly opportunistic animals that exhibit very short life cycles (subannual), and may have a high reproductive output (Kuklinski, Gulliksen et al. 2006; Kuklinski \& Taylor 2006).

Larger stones offer a much more stable environment, because they are harder to overturn, and they host a completely different set of species (Sousa 1979; Wilson 1985; Kuklinski, Gulliksen et al. 2006). In assemblages living on large stones, space is at a premium, and com- petition is prevalent (Barnes \& Kuklinski 2003). This community consists of animals that invest energy in various defense mechanisms or other mechanisms allowing them to secure living space on the stone (Sousa 1979; Wilson 1985).

In the shallow subtidal zone, bryozoans are the most species-rich organisms (Barnes \& Kuklinski 2003). They are also very often the most abundant colonizers of the rocks. However, polychaete (tubicolous) worms usually form more than half of the individuals colonizing stones (Barnes \& Kuklinski 2003; Barnes \& Kuklinski 2004a). Barnacles, colonial ascidians, and occasionally calcarean sponges and demosponges are the other macrofaunal organisms found on shallow subtidal stones (Barnes \& Kuklinski 2004a). Among the six locations investigated on the west coast of Spitsbergen, the species richness ranged from 25 to 41 taxa, and 68 taxa were found in total. The density of macrobiota at these depths ranged from 2720 to 12895 individuals per $\mathrm{m}^{2}$ of the rock surface (Kuklinski \& Barnes 2008).

There is substantial variation in life history investment strategies observed among species found in the shallow subtidal zone (Table 2). Some species are long-lived perennials (e.g., barnacles) that live for many years, reproducing annually. Some bryozoans, after a period of early growth, allocate resources jointly to sexual reproduction and to continued colony growth. There are also examples of short-lived organisms with a single phase of sexual reproduction, which is typically followed by the death of the organism (e.g., the bryozoan H. scutulata). Although species with different life history strategies co-occur, the polar shallow subtidal environment tends to favour particular types of strategies (Table 2). Assemblages on rocks at these depths can comprise many species, but are dominated by just a few (Kuklinski \& Barnes 2005b). Species with unusual life histories (e.g., producing extremely large number of offspring), like the bryozoan $H$. scutulata, can account for over $50 \%$ of the individuals within assemblages (Barnes \& Kuklinski 2003; Kuklinski \& Barnes 2005a, b). The advantage obtained by the unusual life history of this bryozoan means that it is a dominant member of the assemblage, despite being an inferior competitor for space on stones, losing sometimes $70 \%$ of interactions with other organisms (Barnes \& Kuklinski 2003; Kuklinski \& Barnes 2005a, b; Kuklinski \& Taylor 2006). The extreme success of this species is attributed to fast growth and rapid development to maturity, despite little investment in defense (Kuklinski \& Taylor 2006). Colonies of H. scutulata also contain a great number of embryos, which is typical of opportunistic species, and this contributes to their success by increasing the opportunities for the earliest recruitment following a disturbance. For example, the second 
Table 2 Exemplar abundance and interaction data for various cheilostome bryozoans and the barnacle Semibalanus balanoides L. from Hornsund fjord, western Spitsbegen (at a depth of 6 m), indicating various life-history strategies (Barnes \& Kuklinski 2003). There are examples of species that are weak competitors yet recruit in high number (e.g., Harmeria scutulata Busk), and species that recruit in low number yet win the majority of interactions (e.g., Tegella arctica d'Orbigny). Rows list the identities of all species for which interference competition was observed. Columns list the density, total number of competitor species encountered, total number of interspecific encounters, number of wins divided by total number of encounters (WIT) and number of losses divided by total number of encounters (L/T).

\begin{tabular}{|c|c|c|c|c|c|}
\hline Species & $\begin{array}{l}\text { Mean number } \\
\text { recruits } / \mathrm{m}^{2}\end{array}$ & $\begin{array}{l}\text { Number competitors } \\
\text { encountered }\end{array}$ & $\begin{array}{l}\text { Number interspecific } \\
\text { interactions }\end{array}$ & WIT & $L / T$ \\
\hline Tegella retroversa & 46.17 & 3 & 35 & 0.91 & 0.09 \\
\hline Tegella arctica & 83.95 & 4 & 35 & 0.77 & 0.23 \\
\hline Cribrilina annulata & 156.77 & 3 & 10 & 0.70 & 0.30 \\
\hline Tegella armifera & 85.21 & 3 & 16 & 0.62 & 0.187 \\
\hline Cauloramphus intermedius & 67.2 & 4 & 39 & 0.51 & 0.462 \\
\hline Stomachetosella cruenta & 53.95 & 2 & 3 & 0.33 & 0.667 \\
\hline Harmeria scutulata & 3091.3 & 7 & 114 & 0.24 & 0.728 \\
\hline Electra arctica & 27.75 & 1 & 1 & 0 & 1 \\
\hline Semibalanus balanoides & 278.41 & 1 & 2 & 0 & 1 \\
\hline
\end{tabular}

most abundant species (e.g., Cribrilina annulata Fabricius) represents only $5 \%$ of the total fauna.

The shallow subtidal zone is characterized not only by high levels of species richness, but also by a high diversity of different morphotypes for such substrata as stones (Table 1). In this zone the dominating morphological forms are the low-lying encrusters, such as spirorbid polychaetes or bryozoans that grow over the surface of the rocks. These forms enable organisms to better resist the strong currents (Wildish \& Kristmanson 1997). Studies from temperate areas indicate that current velocity can locally modify both biomass and diversity on boulders (Guichard \& Bourget 1998). There are also many organisms present, usually below a depth of $3 \mathrm{~m}$, with arborescent or bushy forms that are characterized by a degree of flexibility. These flexible erect morphs enable organisms to survive strong storms with minimal damage. Bryozoans are the most species-rich group of both lowlying encrusting and erect forms, although hydrozoans and ascidians are also common in some areas. Growing above the bottom is an adaptation that makes organisms more competitive in an environment where space is frequently a limiting factor. Water currents are stronger above the bottom, and therefore bring a richer food supply. Furthermore, a bushy or tree-like morphology helps avoid attacks by certain predators, which cannot handle the stronger currents present at higher levels above the bottom (Wildish \& Kristmanson 1997). Often these three-dimensional erect forms provide habitats for other organisms (e.g., encrusting bryozoans, hydrozoans or foraminiferans). The dynamic nature of the environment exerts an overwhelming impact on the types of biota morphologies that we observe in a given habitat. Yet, with increasing depth, the impact of many physical factors (e.g., wave influence) decreases substantially.
Fragile erect forms such as complex three-dimensional colonies of bryozoans in shallow areas of the Greenland Sea (down to a depth of $50 \mathrm{~m}$ ) are not common. This is attributed to the instability of the shallow subtidal region. Wave action, strong currents and ice scouring are most likely to be responsible for the absence of many fragile erect forms.

Competition for space on the stones in some areas can be very intense (Barnes $\&$ Kuklinski 2003). As indicated by Barnes \& Kuklinski (2004a), competition may be a major factor controlling assemblage structure on stones. Assemblages are organized into hierarchies, where one species is usually dominant (Table 2 ). Often one species outcompetes all of the others, and is able to monopolize the space. Cheilostome bryozoans are involved in most interactions, and the majority of these are in competition with other cheilostome bryozoans. However, ascidians, cirripeds, sponges and polychaetes are also involved in competitive encounters, although much less frequently (Barnes \& Kuklinski 2004a). The probability of an individual organism experiencing competition on a stone varies between localities and depths (from $P=0.01$ to $P=0.18$; Barnes \& Kuklinski 2004a). The chance of encountering a spatial competitor differs over small scales, and can double at sites separated by as little as $10 \mathrm{~m}$ (Barnes \& Kuklinski 2004a). The size of the stone plays an important role affecting interactions among organisms. On rocks smaller than $8 \mathrm{~cm}^{2}$, no competitive interactions were noted, but competitive interactions took place among colonists on almost all stones above $500 \mathrm{~cm}^{2}$ in surface area (Barnes \& Kuklinski 2003). Several other factors also contribute to competition variability on the stones. For example, the density (expressed as a percentage cover of the rock) (Fig. 3) of organisms plays an important role (Barnes \& Kuklinski 2004a). The 

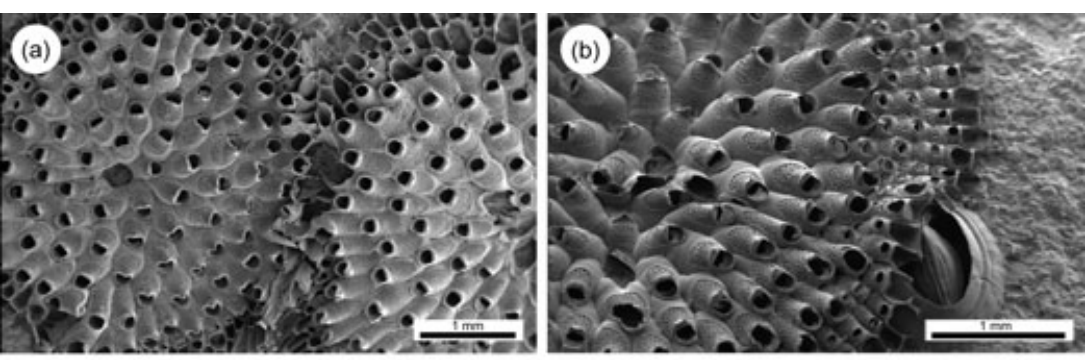

Fig. 5 Examples of interactions. (a) Intraspecific interactions between two individuals of the bryozoan Harmeria scutulata Busk. (b) Interspecific interactions between the bryozoan $H$. scutulata and cirriped Semibalanus balanoides L. (c) Interspecific interaction: Tegella sp. overgrowing Cylindroporella tubulosa Norman. (d) Example of a "stand-off", when two species interact spatially, but neither is overgrowing the other; in this case Tegella sp. and H. scutulata.
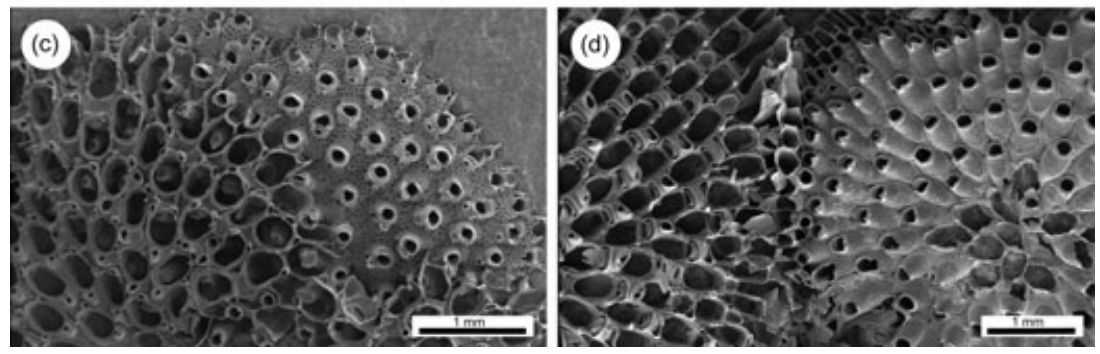

larger the area of rock covered by the biota, the higher the probability of competitive interactions (Barnes $\delta$ Kuklinski 2004a). Among biological factors, taxonomical membership both at the class and phylum level explains the majority of variability in competitive performance (Barnes \& Kuklinski 2004b). The best competitors are ascidians, then demosponges (but both are very rare) and bryozoans of the genus Tegella, whereas poor competitors are thinly calcified cheilostome bryozoans (e.g., Celleporella hyalina L.), then cyclostome bryozoans, barnacles, hydroid cnidarians and tubicolous polychaetes (all weak competitors and common) (Barnes \& Kuklinski 2004b). In general, at most locations, a larger proportion of interactions on stones are intraspecific (Fig. 5a; Barnes \& Kuklinski 2003; Barnes \& Kuklinski 2005b). However, the proportion of interspecific (Fig. 5b-d) competition is very variable, and ranges from 20.8 to $97.2 \%$, or from communities with almost no intraspecific encounters to those heavily dominated by them (Barnes $\&$ Kuklinski 2004a). Greenland Sea assemblages comprise a few pioneer species that tend to lose all competitive interactions for space, and a few dominant species that win all competitive encounters (Barnes \& Kuklinski 2004b) (Table 2). As previously discussed, physical disturbance can have a profound effect on community structure, and also affects competition (Barnes \& Kuklinski 2005b). For instance, in places where ice has recently scoured the sea bottom, little colonization occurs, interactions are rare, and pioneers are the most abundant, so that most of the encounters are intraspecific (Barnes \& Kuklinski 2005b).

There are virtually no data on the influence of predation on stone assemblages at these depths. Yet there are a large number of predators present in the area that normally feed on rocky-bottom fauna (Gulliksen et al. 1999).
These include sea urchins, ophiuroids, polyplacophorans and gastropods (Kuklinski, pers. obs.). For example, the dominant Arctic sea urchin Strongylocentrotus droebachiensis (Müller) is known to eat macroalgae, benthic diatoms and small invertebrates such as ascidians, polychaetes and young mussels (e.g., Himmelman \& Steele 1971). Arctic sites that are densely inhabited by this species have a lower diversity of macrofauna on stones compared with nearby locations (Kuklinski \& Barnes 2005a). All the above mentioned predators are potentially capable of modifying their local stone-dwelling assemblages. However, their influence has been not studied to date.

\section{Deep subtidal zone}

There is much less knowledge about stone fauna from deeper parts of the Greenland Sea than from both the intertidal and shallow subtidal zones. This is mostly attributed to the lower accessibility of these areas. So far, only two studies have focused on the fauna colonizing stones from deeper parts of the shelf (Kuklinski $\&$ Bader 2007a, b). These studies investigated the East Greenland and west Spitsbergen shelves. On about a hundred rocks from a polynya (open water area in an otherwise icecovered sea) in the vicinity of the East Greenland coast, 113 taxa were recognized, including 58 genera, 38 families, 12 orders and eight phyla (Kuklinski $\&$ Bader 2007a, b) (Table 1). Bryozoans are the most species-rich, but foraminiferans, polychaetes, sponges, hydrozoans, balanomorphs, anthozoans and ascidians are also present. The most abundant group of organisms are foraminiferans, bryozoans and polychaetes, whereas the least abundant are anthozoans and ascidians (Kuklinski $\delta$ Bader 2007b). However, there seems to be variation both 
in species composition and total species richness among Greenland Sea locations at these depths. For example, samples from East Greenland were found to be one third richer in bryozoan taxa than samples from west Spitsbergen (Fig. 6; Table 1). Both localities are at the same latitude (roughly $79^{\circ} \mathrm{N}$ ) and have a similar depth range (75-260 m), but differ in environmental conditions such as hydrology, distance from the land (and therefore influence of terrigenous material, e.g., sediment discharge) and level of ice cover (Kuklinski \& Bader 2007a). This contrast in abiotic parameters was reflected in species composition and dominance structure, which differed to a large extent between sites. There was a much higher proportion of Arctic endemic species at the East Greenland site than at west Spitsbergen (Table 1). However, there were no significant differences in the mean values of species richness, diversity and abundance between these two locations (Kuklinski \& Bader 2007a). The variations observed in assemblages between locations are most likely to have been caused by the interplay of many factors, and any explanations remain speculative.

On a scale from metres to a few kilometres, the surface area of stones was found to have a significant influence on faunal species richness and abundance. Total recruits and species numbers increase with rock size at these depths (e.g., rocks of surface area ca. $100 \mathrm{~cm}^{2}$ supported 61-166 recruits and 10-12 species, whereas rocks of surface area above $500 \mathrm{~cm}^{2}$ supported 61-372 recruits and 6-27 species [Kuklinski \& Bader 2007b]). There is also a strong relationship between the numbers of species and individuals on the rocks: the more recruits there are, the more species there are (Kuklinski \& Bader 2007b).

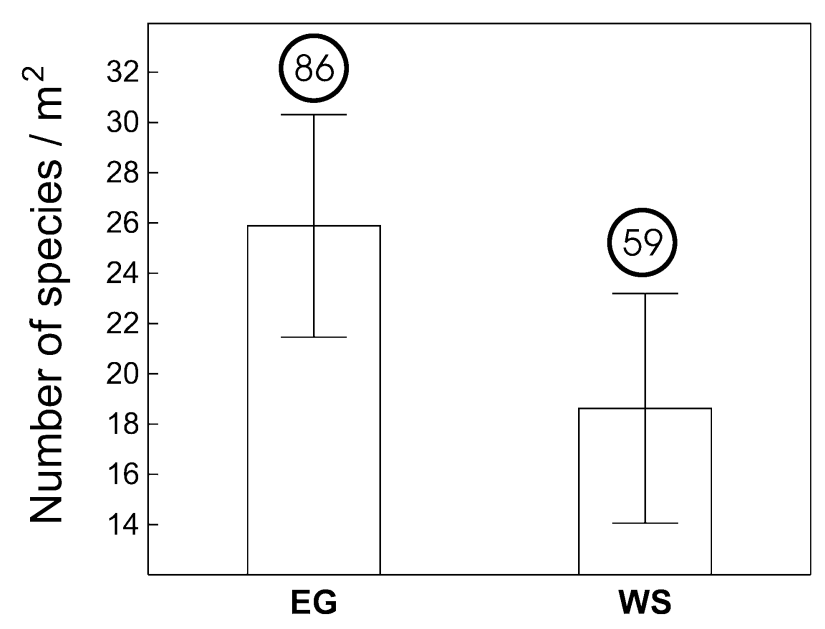

Fig. 6 Mean values \pm standard error and total number (in circles) of bryozoan species from ca. 100 stones collected from several sampling stations located on East Greenland (EG) and western Spitsbergen (WS) shelves. (After Kuklinski \& Bader 2007a.)
Typically, deeper parts of the shelf contain soft homogeneous sediments (Zaborska et al. 2006). Only the presence of hard substrata provided by stones allows hard-bottom organisms to occur at these depths. Rocks are usually partly buried in the fine sediment. This leads to the colonization of just the top parts. To our knowledge, most stone-encrusting organisms are suspension feeders (Barnes \& Ruppert 1994). Therefore, the reduced quantities of food reaching deeper water may also promote the colonization of upper surfaces, as growing here makes the capture of falling food particles easier.

Flat encrusting morphological structures are the most abundant forms on the stones, both in terms of species and individual numbers. Yet at some locations (e.g., East Greenland) bryozoans, hydrozoans and occasionally ascidians exhibit a high proportion of both rigid and flexible erect forms (Kuklinski \& Bader 2007b). The occurrence of numerous fragile erect rigid forms is often an indication of the lack of frequent dynamic physical disturbances (Kuklinski et al. 2005; Kuklinski \& Bader $2007 b)$. Stones must remain stable for some reasonable period of time (no data on the growth rates of Arctic encrusting organisms exist) to allow these forms to develop. Therefore, the presence of fragile erect forms can be attributed to substratum stability and longevity (Wilson 1987; Lidgard 1990).

As in the shallow subtidal zone, erect forms of invertebrates growing on the stones often create additional three-dimensional structures. It is believed that at these depths such growth forms can provide a refuge from being buried during episodes of resuspension. Once an organism is established as an erect form, it creates extra ecological niches that are occupied by species present in very low numbers or absent on the stone itself. Therefore, among organisms present on stones, it is possible to distinguish: (1) primary space colonizers, which are restricted to stones; (2) secondary space colonizers, which prefer the three-dimensional structures provided by erect morphs of organisms such as erect bryozoans; and (3) generalists with a broad spectrum of space colonization, including both rock surfaces and three-dimensional structures (Kuklinski \& Bader 2007b). Within all of these categories, bryozoans constitute the most species-rich group (Kuklinski \& Bader 2007a, b).

Competition is low in the deep subtidal zone. Data gathered so far indicate that competition for space is of minor importance. This is shown by the low number of interactions and the abundance of free space available on the stones from these depths (Kuklinski \& Bader 2007a). The lack of space restriction on rocks also suggests that most of the competitive encounters are probably random events, rather than real fights for space. Investigation by Kuklinski \& Bader (2007a) revealed that among all the 
organisms present on stones, only $3 \%$ of them were involved in any competitive contact. The great majority of these interactions were interspecific. Most encounters involved bryozoans, but polychaetes, sponges and foraminiferans were also sometimes encountered. When present, sponges always outcompeted other organisms. Other factors that influence interactions at these depths are still not fully understood. At present, we know that rock size has little influence on the number of interactions; however, with increasing numbers of species on the rock, both the number of inter- and intraspecific interactions increase (Kuklinski \& Bader 2007b). The abundance of organisms or area of space occupied by biota on the rock does not influence the number of interactions. Current research suggests that organism identity is very important for interaction outcome. As observed by Kuklinski \& Bader (2007a), interactions show similar results to those from lower latitudes (e.g., most encounters are among encrusting bryozoans, and sponges are superior competitors) or shallows in the Greenland Sea (Barnes 2002; Barnes \& Kuklinski 2004a, b; Kuklinski \& Bader 2007a).

As in the shallow subtidal zone, there are a large number of predators present in the area that are able to feed on rocky bottom fauna (Piepenburg et al. 1996). For example, these include sea stars, crustaceans and gastropods (Kuklinski, pers. obs.). Yet, nothing is known about the influence of predation on the biota of stones from these depths.

\section{Glacial bays}

Bays in the vicinity of tidal glaciers (often in the inner fjordic area) are very peculiar environments. The water depths of glacial bays range from 5 to $240 \mathrm{~m}$. The sea bottom in these areas is dominated by unconsolidated, labile sediments, and it experiences an extreme sediment load, especially during summer, reaching up to $800 \mathrm{mg} \mathrm{dm}^{-3}$ (Elverhoi et al. 1983). Icebergs and growlers (smaller pieces of glacier ice, not extending $1 \mathrm{~m}$ above the sea surface) are observed in these areas the whole year round (Fig. 2d). They are the source of the stones that are often the only hard substrata available in this area (Dowdeswell \& Forsberg 1992). Here, stones are distributed in a patchy pattern, and organisms colonizing them create "faunistic islands" (Kuklinski 2005). There seems to be a positive correlation between the number of stones and the density of the organisms inhabiting the faunal island (Dale et al. 1989). Species, if present, colonizing these stones are usually characterized by erect flexible growth forms. Through their flexibility and bending, they are able to use ambient currents to shed sediment. Also, many of the organisms possess specialized organs, for example avicularia equipped with long seta in bryozoans, which might enable the organism to sweep the sediment off their surfaces.

Both species diversity and abundance are greatly reduced compared with stones from other parts of the polar ecosystem (Kuklinski 2005). The seasonally heavy sedimentation in glacial bays occurring in summer is most likely to be responsible for this reduction. Also, heavy sedimentation might, for example, coincide with the reproductive period, thereby enabling species to settle and develop on a stone (see Taylor $\&$ Wilson 2003). Once larvae are released into the water column some of them can travel long distances (e.g., Scheltema 1986). Yet, the lack of suitable firm substratum because of the heavy load of sedimentation might prevent them from settlement. Furthermore, a high sedimentation rate can clog feeding and respiratory organs, leading to increased mortality (Moore 1977). In general, stones covered with fine sediment tend to be unpopulated (Kuklinski, pers. obs.).

\section{Conclusions and future challenges}

Information gathered to date has revealed a few patterns of faunal assemblage development on stones in the Greenland Sea. There is low biodiversity and a lack of habitat specialist species on stones in the intertidal zone (Table 1), which is probably caused by the extreme level of disturbance in this zone and the evolutionary youth of the Arctic biota (Kuklinski \& Barnes 2008). Below the intertidal zone all parameters of stone assemblages change dramatically. There is a large increase in species diversity, as well as in morphological forms and abundance. Faunal assemblages here exhibit a broad morphological diversity, ranging from small encrusting unicellular foraminiferans to diverse erect forms. In the shallow subtidal zone an increase in the diversity of species and morphological forms is most likely attributable to the increases in environmental stability and food availability. With increasing depth, a number of physical forces (e.g., icebergs and wave action) decrease in intensity. Therefore, rocks immersed in deeper waters experience less disturbance and support a greater diversity of morphological forms. For example, delicate, arborescent, calcareous forms are only present in deeper, less disturbed sections of the ecosystem. The other pattern that is evident on the stones in the Greenland Sea is a decrease in the abundance of encrusting organisms, as well as in the intensity of competitive interactions, with depth. These two patterns seem to be linked together and depend upon food availability. In contrast, the overall species richness in the subtidal zone seems to increase with depth. A schematic model of factors affecting the Greenland Sea stone assemblages is presented in Fig. 7. 


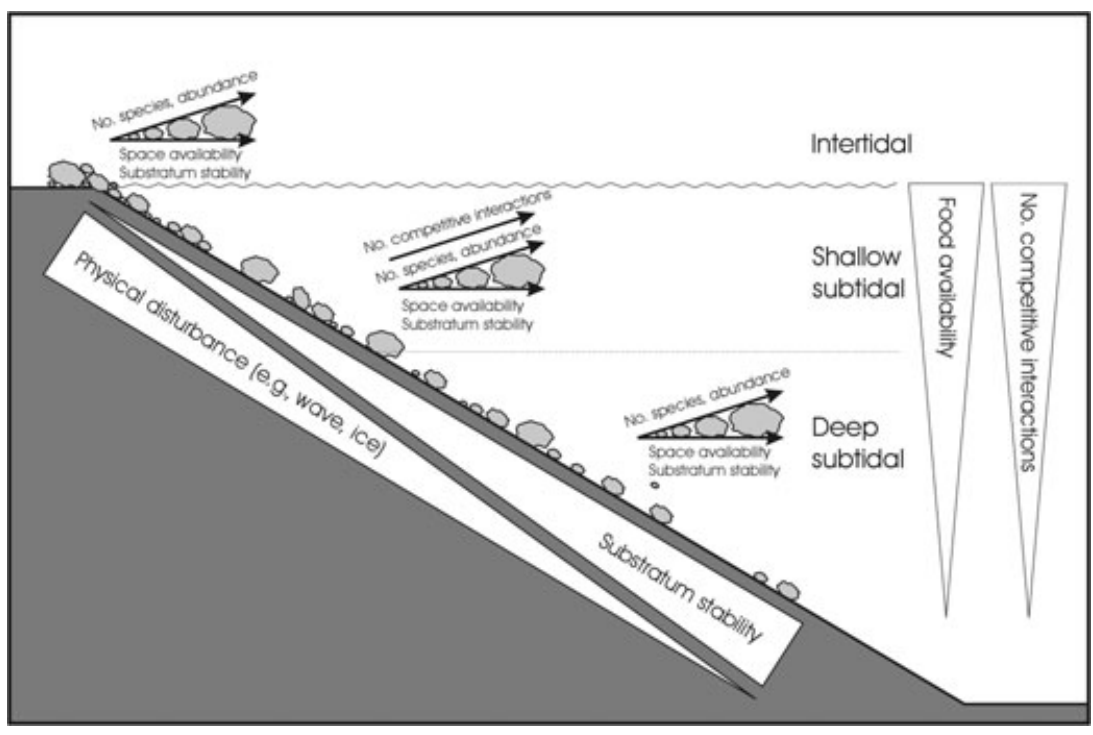

Fig. 7 Schematic model of factors affecting the Greenland Sea stone assemblages at different depth zones.
The structure of Arctic stone assemblages resembles those of the Antarctic. In the Antarctic intertidal and shallow subtidal zones, as in the Arctic, spirorbid polychaetes and cheilostomate bryozoans are the dominating encrusting organisms, both in terms of species richness and abundance (Barnes \&Arnold 1999; Barnes 2000). There is also a high proportion of intraspecific interactions in both polar regions (see Barnes 2000). When Arctic stone assemblages are compared with temperate and tropical shores, lower diversity is evident in the Arctic encrusting fauna. Whereas there are commonly between three and six phyla in the intertidal and shallow subtidal zones in non-polar waters, in the Arctic there are usually only two phyla represented (polychaetes and bryozoans [Barnes 2000]). The reduction in the number of encrusting species with latitude also has an effect on the pattern of the faunal interactions. For example, the intensity of interspecific interactions is lower in the Arctic compared with temperate or tropical areas (Barnes 2000; Barnes 8 Kuklinski 2003).

The current rapid climatic changes in the Arctic are very likely to influence stone assemblages (Barnes $\delta$ Kuklinski 2003; Kuklinski \& Bader 2007a). The warming of the Arctic water masses can be expected to result in a compositional change in species. Species with narrow Arctic distributions may be replaced by Boreal species, or by species able to cope with a broad range of sea-water temperatures (Kuklinski \& Bader 2007a). The real Arctic endemic species are at risk of extinction (Kuklinski $\delta$ Bader 2007a).

The predicted sea temperature rises in the Arctic could dramatically change the disturbance regime, moderating the floating ice level and ice cover, as well as wave action. The initial influence of warming is likely to increase the volume of floating ice, as a result of glacier collapse (Doake \& Vaughan 1991), but in a later phase the volume of floating ice will decrease because of reduced winter sea-surface freezing. This should drastically increase the time for which Arctic intertidal and shallow subtidal sites are available for colonization, thereby increasing the diversity and abundance of biota. In a later stage, the most competitively superior species could colonize a large proportion of the space on stones. It is therefore expected that in the short term reduced disturbance will lower the abundance of the poorest competitors. In the medium term, with ice disappearing in the Arctic, a temporary increase in patchiness, diversity and interspecific competition will occur as the level of disturbance decreases (Barnes \& Kuklinski 2005b). Ultimately, some species of bryozoans and various ascidians are likely to occupy all of the available space, causing diversity to decline and intraspecific competition to predominate in encrusting Arctic stone assemblages (Barnes \& Kuklinski 2005b).

The changes in stone-dwelling faunal assemblages caused by global warming are ongoing. Several studies have shown that sessile benthic assemblages are good indicators of long-term ecosystem change (WlodarskaKowalczuk \& Weslawski 2001; Beuchel et al. 2006). Palaeontologists have used fossilized stone-encrusting assemblages to reconstruct the environmental conditions of the Upper Ordovician (Wilson 1985). Polar patterns of intraspecific competition are suggested to be a powerful signal of the extent of biological responses to polar ocean warming and glacial collapses (Barnes \& Kuklinski 2005b). They are, for example, more hierarchical in polar regions than elsewhere at lower latitudes (Barnes \& Kuklinski 2005b). Therefore, because of the higher sensitivity of shallow water areas to observed climate changes, our 
relatively detailed knowledge about biodiversity and ecological processes on the stones, and their accessibility, these assemblages seem to be a good surrogate measure of faunal responses to climate change (Barnes \& Kuklinski 2005b).

Our knowledge of stone assemblages is still limited to small geographical areas. Most of the studies reviewed here focus on the western coast of Spitsbergen, which might not be representative of the rest of the Arctic. A greater sampling effort is suggested, to cover greater depths as well as broader areas, so that the diversity of the Greenland Sea and the Actic as a whole is better represented. Such studies may lead to an improved understanding of the forces driving the diversity of the encrusting biota. For example, considerable patchiness in biodiversity and ecological processes from the intertidal to deep subtidal zones are evident. Even in deeper areas of the Greenland Sea continental shelf, which seem to be homogeneous, quite stable and devoid of large disturbances caused by strong winter storms, ice scour and wave action, etc., patchiness in species richness and abundance is observed (e.g., Kuklinski \& Bader 2007a, b). Knowledge of the long-term availability of stones, as well as of their supply by glaciers in specific areas, especially in deeper soft-bottom subtidal zones, may be critical for our understanding of these ecological processes. In such areas, the distances between stones may be so far that they are beyond the dispersal capabilities of the organisms that would normally colonize them. The majority of Arctic encrusting organisms have lecitotrophic larvae (Kuklinski, Gulliksen et al. 2006) that stay in the water for hours rather than days, which means that their dispersal is limited to short distances. Also, broad investigations, including studies of the shape, complexity and mineralogy of rocks or food-quality influence on stone assemblages, should increase our knowledge about the ecology of this biota.

This review has illustrated many different adaptations of encrusting Arctic biota to the changes in environmental dynamics usually associated with processes related to depth (e.g., wave action, ice scour and food availability). Studying these adaptations may lead to an understanding of the influence of climate change on faunal assemblages or even evolutionary traits of these organisms. Yet, surprisingly, in the majority of cases we know nothing or very little about the life histories or biogeographical origins of the organisms colonizing the rocks. This large gap in our knowledge should be quickly filled before the Arctic organisms begin to vanish.

A large number of studies in recent years, both from polar and lower latitudes, indicate the great potential of rock-encrusting faunas for studying benthic ecological processes (e.g., Sousa 1979; Wilson 1985, 1987;
McGuinnes 1987; Guichard \& Bourget 1998; Barnes 2002; Taylor \& Wilson 2003; Kuklinski, Barnes et al. 2006). This review can serve as a baseline study to accelerate further studies in the most rapidly changing environment: the Arctic.

\section{Acknowledgements}

I would like to thank Suzanne Williams, Paul Taylor, Mark Wilson and three anonymous referees for comments and corrections leading to the improvement of the manuscript. The study has been completed thanks to funding provided by a DYNARC grant from the European Commission's programme Structuring the European Research Area, and a grant from the Polish Ministry of Science and Higher Education (N N304 270434).

\section{References}

Arntz W.E., Brey T. \& Gallardo V.A. 1994. Antarctic zoobenthos. Oceanography and Marine Biology: an Annual Review 32, 251-303.

Barnes D.K.A. 1999. The influence of ice on polar nearshore benthos. Journal of the Marine Biology Association of the United Kingdom 79, 401-407.

Barnes D.K.A. 2000. Diversity, recruitment and competition on island shores at south-polar localities compared with lower latitudes: encrusting community examples. Hydrobiologia 440, 37-44.

Barnes D.K.A. 2002. Polarization of competition increases with latitude. Proceedings of the Royal Society of London B 269, 2061-2069.

Barnes D.K.A. \& Arnold R.J. 1999. Possible latitudinal clines in Antarctic intertidal and subtidal zone communities encrusting ephemeral hard substrata. Journal of Biogeography 26, 207-213.

Barnes D.K.A. \& Conlan K. 2007. Disturbance, colonization and development of Antarctic benthic communities. Philosophical Transactions of the Royal Society B 362, 11-38.

Barnes D.K.A. \& Kuklinski P. 2003. High polar spatial competition: extreme hierarchies at extreme latitude. Marine Ecology Progress Series 259, 17-28.

Barnes D.K.A. \& Kuklinski P. 2004a. Variability of competition at $10^{1}, 10^{3}, 10^{5}$, and $10^{6} \mathrm{~m}$ scales: encrusting community patterns. Marine Biology 145, 361-372.

Barnes D.K.A. \& Kuklinski P. 2004b. Scale-dependent variation in competitive ability among encrusting Arctic species. Marine Ecology Progress Series 275, 21-31.

Barnes D.K.A. \& Kuklinski P. 2005a. Low colonisation on artificial substrata in Arctic Spitsbergen. Polar Biology 29, 65-69.

Barnes D.K.A. \& Kuklinski P. 2005b. Bipolar patterns of intraspecific competition in bryozoans. Marine Ecology Progress Series 285, 75-87.

Barnes R.D. \& Ruppert E.E. 1994. Invertebrate zoology. Philadelphia: Saunders College Publishing. 
Begon M., Harper J.L. \& Townsend C.R. 1996. Ecology: individuals, populations and communities. Oxford: Blackwell Science.

Berge J., Johnsen G., Nilsen F., Gulliksen B. \& Slagstad D. 2005. Ocean temperature oscillations enable reappearance of blue mussel Mytilus edulis in Svalbard after $1000 \mathrm{yr}$ absence. Marine Ecology Progress Series 303, 167-175.

Beuchel F., Gulliksen B. \& Carroll M.L. 2006. Long-term patterns of rocky bottom macrobenthic community structure in an Arctic fjord (Kongsfjorden, Svalbard) in relation to climate variability (1980-2003). Journal of Marine Systems 63, 35-48.

Booth B.C. \& Smith W.O. 1997. Autotrophic flagellates and diatoms in the North Water Polynya, Greenland: summer 1993. Journal of Marine Systems 10, 241-261.

Clarke A. \& Lidgard S. 2000. Spatial patterns of diversity in the sea: bryozoan species richness in the North Atlantic. Journal of Animal Ecology 69, 799-814.

Connell J.H. 1978. Diversity in tropical rain forests and coral reefs. Science 199, 1302-1310.

Cottier F.R., Tverberg V., Inall M.E., Svendsen H., Nilsen F. \& Griffiths C. 2005. Water mass modification in an Arctic fjord through cross-shelf exchange: the seasonal hydrography of Kongsfjord, Svalbard. Journal of Geophysical Research-Oceans 110, article no. C12005, doi: 10.1029/ 2004JC002757.

Dale J.E., Aitken A.E., Gilbert R. \& Risk M.J. 1989. Macrofauna of Canadian Arctic fjords. Marine Geology 85, 331-358.

Dayton P.K. 1990. Polar benthos. In W.O. Smith (ed.): Polar oceanography. Pp. 631-685. London: Academic Press.

Doake C.S.M. \& Vaughan D.G. 1991. Rapid disintegration of the Wordie ice shelf in response to atmospheric warming. Nature 350, 328-330.

Dowdeswell J.A., Elverhoi A. \& Spielhagen R. 1998. Glacimarine sedimentary processes and facies on the Polar North Atlantic margins. Quaternary Science Review 17, 243-272.

Dowdeswell J.A. \& Forsberg C.F. 1992. The size and frequency of icebergs and bergy bits derived from tidewater glaciers in Kongsfjorden, northwest Spitsbergen. Polar Research 11, 81-91.

Dunton K.H. 1992. Arctic biogeography: the paradox of the marine benthic fauna and flora. Trends in Ecology and Evolution 7, 183-189.

Elverhoi A., Lonne O. \& Seland R. 1983. Glaciomarine sedimentation in a modern fjord environment, Spitsbergen. Polar Research 1, 127-149.

Gilbert, R. 1984. The movement of gravel by the alga Fucus vesiculosus (L.) on an Arctic intertidal flat. Journal of Sedimentary Petrology 54, 463-468.

Guichard F. \& Bourget E. 1998. Topographic heterogeneity, hydrodynamics, and benthic community structure: a scale-dependent cascade. Marine Ecology Progress Series 171, 59-70.

Gulliksen B., Palerud R., Brattegaard T. \& Sneli J. 1999. Distribution of marine benthic macro-organisms at Svalbard (including Bear Island) and Jan Mayen. Trondheim: Directorate for Nature Management.

Himmelman J.H \& Steele D.H. 1971. Foods and predators of the green sea urchin, Strongylocentrotus droebachiensis, in Newfoundland waters. Marine Biology 9, 315-322.

Hop H., Pearson T., Hegseth E.N., Kovacs K.M., Wiencke C., Kwasniewski S., Eiane K., Mehlum F., Gulliksen B., Włodarska-Kowalczuk M., Lydersen C., Weslawski J.M., Cochrane S., Gabrielsen G.W., Leakey R., Lønne O.J., Zajaczkowski M., Falk-Petersen S., Kendall M., Wängberg S.A., Bischof K., Voronkov A.Y., Kovaltchouk N.A., Wiktor J., Poltermann M., Prisco A., Papucci C. \& Gerland S. 2002. The marine ecosystem of Kongsfjorden, Svalbard. Polar Research 21, 167-208.

Kuklinski P. 2002. Bryozoa of the High Arctic fjord-a preliminary study. In P.N. Wyse Jackson et al. (eds.): Bryozoan studies 2001. Pp. 175-183. Lisse, the Netherlands: Swets \& Zeitlinger.

Kuklinski P. 2005. Bryozoan mode of life in the High Arctic dynamic fjordic environment. In H.I. Moyano et al. (eds.): Bryozoan studies 2004. Pp. 153-161. London: A.A. Balkema Publishers.

Kuklinski P. \& Bader B. 2007a. Comparison of bryozoan assemblages from two contrasting Arctic shelf regions. Estuarine, Coastal and Shelf Science 73, 835-843.

Kuklinski P. \& Bader B. 2007b. Diversity, structure and interactions of encrusting lithophillic macrofaunal assemblages from Belgica Bank, East Greenland. Polar Biology 30, 709-717.

Kuklinski P. \& Barnes D.K.A. 2005a. Bryodiversity on coastal boulders at Spitsbergen. In H.I. Moyano et al. (eds.): Bryozoan studies 2004. Pp. 161-172. London: A.A. Balkema Publishers.

Kuklinski P. \& Barnes D.K.A. 2005b. Microhabitat diversity of Svalbard Bryozoa. Journal of Natural History 39, 539-554.

Kuklinski P. \& Barnes D.K.A. 2008. Structure of intertidal and subtidal assemblages in Arctic vs temperate boulder shores. Polish Polar Research 29, 203-218.

Kuklinski P., Barnes D.K.A. \& Taylor P.D. 2006. Latitudinal patterns of diversity and abundance in North Atlantic intertidal boulder-fields. Marine Biology 149, 1577-1583.

Kuklinski P., Gulliksen B., Lønne O.J. \& Weslawski J.M. 2005. Composition of bryozoan assemblages related to depth in Svalbard fjords and sounds. Polar Biology 28, 619-630.

Kuklinski P., Gulliksen B., Lønne O.J. \& Weslawski J.M. 2006. Substratum as a structuring influence on assemblages of Arctic bryozoans. Polar Biology 29, 652-661.

Kuklinski P. \& Taylor P.D. 2006. Unique life history strategy in a successful Arctic bryozoan, Harmeria scutulata. Journal of the Marine Biology Association of the United Kingdom 86, 1035-1046.

Lidgard S. 1990. Growth in encrusting cheilostome bryozoans: II. Circum-Atlantic distribution patterns. Paleobiology 16, 304-321.

Loeng H. 1991. Features of the physical oceanographic conditions of the Barents Sea. Polar Research 10, 5-18. 
Mayer M. \& Piepenburg D. 1996. Epibenthic community patterns on the continental slope off East Greenland at $75^{\circ}$ N. Marine Ecology Progress Series 143, 151-164.

McGuinnes K.A. 1987. Disturbance and organisms on boulder. I. Patterns in the environment and the community. Oecologia 71, 409-419.

Moore P.G. 1977. Inorganic particulate suspensions in the sea and their effects on marine animals. Oceanography and Marine Biology: an Annual Review 15, 225-363.

Oschmann W. 1990. Dropstones-rocky mini-islands in high-latitude pelagic soft substrate environments. Senckenbergiana Maritima 21, 55-75.

Piepenburg D., Chernova N.V., von Dorrien C.F., Gutt J., Neyelov A.V., Rachor E., Saldanha L. \& Schmid M.K. 1996. Megabenthic commmunities in the waters around Svalbard. Polar Biology 16, 431-446.

Reineck H.E. \& Singh I.B. 1975. Depositional sedimentary environments. Heidelberg: Springer.

Ronowicz M. 2005. Species diversity of Arctic gravel beach: case study for species poor habitats. Polish Polar Research 26, 287-297.

Ronowicz M. 2007. Benthic hydroids (Cnidaria: Hydrozoa) from Svalbard waters-biodiversity and distribution. Journal of the Marine Biological Association of the United Kingdom 87, 1089-1094.

Scheltema R.S. 1986. Long-distance dispersal of planktonic larvae of shoal-water benthic invertebrates among central Pacific islands. Bulletin of Marine Science 39, 241256.
Schiermeier Q. 2007. The new face of the Arctic. Nature 446, 133-135.

Sousa W.P. 1979. Disturbance in marine intertidal boulder fields: the nonequilibrium maintenance of species diversity. Ecology 60, 1225-1239.

Szymelfenig M., Kwasniewski S. \& Weslawski J.M. 1995. Intertidal zone of Svalbard 2. Meiobenthos density and abundance. Polar Biology 15, 137-141.

Taylor P.D. \& Wilson M.A. 2003. Palaeoecology and evolution of marine hard substrate communities. Earth-Science Reviews 62, 1-103.

Weslawski J.M., Wiktor J., Zajączkowski M. \& Swerpel S. 1993. Intertidal zone of Svalbard 1. Macroorganism distribution and biomass. Polar Biology 13, 73-79.

Wildish D. \& Kristmanson D. 1997. Benthic suspension feeders and flow. Cambridge: Cambridge University Press.

Wilson M.A. 1985. Disturbance and ecologic succession in an Upper Ordovician cobble-dwelling hardground fauna. Science 228, 575-577.

Wilson M.A. 1987. Ecological dynamics on pebbles, cobbles, and boulders. Palaios 2, 594-599.

Wlodarska-Kowalczuk M. \& Weslawski J.M. 2001. Impact of climate warming on Arctic benthic biodiversity: a case study of two Arctic glacial bays. Climate Research 18, 127-132.

Zaborska A., Pempkowiak J. \& Papucci C. 2006. Some sediment characteristics and sedimentation rates in an Arctic Fjord (Kongsfjorden, Svalbard). Annual Environmental Protection 8, 79-96. 